\title{
Synthesis and Biological Evaluation of Cyclopropyl Analogues of Fosmidomycin as Potent $P$. falciparum Growth Inhibitors.
}

Vincent Devreux, Jochen Wiesner, Jan L. Goeman, Johan Van der Eycken, Hassan Jomaa, and Serge Van Calenbergh ${ }^{*}$

\section{SUPPORTING INFORMATION - CONTENTS}

Experimental section and analytical data for intermediates and final products $\quad$ S2

HPLC purity of inhibitors

S8

Determination of relative configuration of cyclopropane rings

S9 


\section{Experimental Procedures}

\section{Dibenzyl 3R-hydroxy-4-(p-toluenesulfonyloxy)butanephosphonate (9b)}

Was prepared according to the same procedure as 9a using $(R)$-glycidyl tosylate yielding a thick grey-white oil $(52 \%)$.

$[\alpha]_{\mathrm{D}}=+2.3\left(\mathrm{c}=1.15\right.$ in $\left.\mathrm{CHCl}_{3}\right) ;{ }^{1} \mathrm{H}$ NMR $\left(300.13 \mathrm{MHz}, \mathrm{CDCl}_{3}\right) \delta 1.57-1.97(4 \mathrm{H}, \mathrm{m}), 2.42(3 \mathrm{H}, \mathrm{s})$, 3.77-3.92 $(3 \mathrm{H}, \mathrm{m}), 4.91(1 \mathrm{H}, \mathrm{dd}, J=11.9$ and $8.4 \mathrm{~Hz}), 4.92(1 \mathrm{H}, \mathrm{dd}, J=11.9$ and $8.3 \mathrm{~Hz}), 5.01(2 \mathrm{H}$, dd, 11.9 and 9.2), 7.29-7.33 (12H, m), $7.76(2 \mathrm{H}, \mathrm{d}, J=8.3 \mathrm{~Hz}) ;{ }^{13} \mathrm{C} \mathrm{NMR}\left(75.47 \mathrm{MHz}, \mathrm{CDCl}_{3}\right)$ $\delta 21.6\left(\mathrm{CH}_{3}\right), 21.9\left(\mathrm{P}_{-} \mathrm{CH}_{2},{ }^{1} J_{P C}=142.2 \mathrm{~Hz}\right), 25.8\left(-\mathrm{CH}_{2},{ }^{2} J_{P C}=4.9 \mathrm{~Hz}\right), 67.4\left(-\mathrm{OCH}_{2},{ }^{2} J_{P C}=6.0\right.$ $\mathrm{Hz}), 68.8\left(-\mathrm{OCH},{ }^{3} J_{P C}=13.2 \mathrm{~Hz}\right), 72.9\left(-\mathrm{OCH}_{2}\right), 128.0(=\mathrm{CH}), 128.5(=\mathrm{CH}), 128.6(=\mathrm{CH}), 129.9$ $(=\mathrm{CH}), 132.7(=\mathrm{CH}), 136.2\left(=\mathrm{C},{ }^{3} J_{P C}=5.5 \mathrm{~Hz}\right), 145.0(=\mathrm{C}) ;{ }^{31} \mathrm{P} \mathrm{NMR}\left(121.50 \mathrm{MHz}, \mathrm{CDCl}_{3}\right)$ $\delta 33.6 ;$ ESMS $m / z, 505\left([\mathrm{M}]+\mathrm{H}^{+}\right)$.

\section{Dibenzyl 1-phenyl-3-hydroxy-4-( $p$-toluenesulfonyloxy)butanephosphonate (16)}

Was prepared using the same procedure as 9a, but was additionally crystallised from $\mathrm{Et}_{2} \mathrm{O}$ giving white crystals as a $1 / 1$ mixture of syn/anti stereoisomers $(81 \%)$.

${ }^{1} \mathrm{H}$ NMR $\left(300.13 \mathrm{MHz}, \mathrm{CDCl}_{3}\right) \delta 2.00-2.32(4 \mathrm{H}, \mathrm{m}), 2.42(3 \mathrm{H}, \mathrm{s}), 2.43(3 \mathrm{H}, \mathrm{s}), 3.26(1 \mathrm{H}, \mathrm{dt}, J=$ 22.5 and $7.5 \mathrm{~Hz}), 3.40(1 \mathrm{H}$, ddd, 22.4, 10.7 and $4.7 \mathrm{~Hz}), 3.50(1 \mathrm{H}, \mathrm{m}), 3.77(1 \mathrm{H}, \mathrm{dd}, J=10.2$ and $6.2 \mathrm{~Hz}), 3.80(1 \mathrm{H}, \mathrm{dd}, J=10.2$ and $6.6 \mathrm{~Hz}), 3.87(1 \mathrm{H}, \mathrm{dd}, J=10.0$ and $3.6 \mathrm{~Hz}), 3.88(1 \mathrm{H}, \mathrm{dd}, J=$ 10.1 and $3.6 \mathrm{~Hz}), 3.96(1 \mathrm{H}, \mathrm{m}), 4.60(1 \mathrm{H}, \mathrm{dd}, J=11.8$ and $8.4 \mathrm{~Hz}), 4.61(1 \mathrm{H}, \mathrm{dd}, J=11.8$ and 8.4 $\mathrm{Hz}), 4.77(2 \mathrm{H}, \mathrm{dd}, J=11.7$ and $7.4 \mathrm{~Hz}), 4.87(1 \mathrm{H}, \mathrm{dd}, 11.7$ and $7.9 \mathrm{~Hz}), 4.87(1 \mathrm{H}, \mathrm{dd}, J=11.7$ and $7.8 \mathrm{~Hz}), 4.89(1 \mathrm{H}, \mathrm{dd}, J=11.7$ and $7.8 \mathrm{~Hz}), 4.97(1 \mathrm{H}, \mathrm{dd}, J=11.8$ and $9.1 \mathrm{~Hz}), 4.98(1 \mathrm{H}, \mathrm{dd}, J=$ 11.8 and $9.4 \mathrm{~Hz}), 7.15-7.35(34 \mathrm{H}, \mathrm{m}), 7.77(4 \mathrm{H}, \mathrm{d}, J=8.3 \mathrm{~Hz}) ;{ }^{13} \mathrm{C} \mathrm{NMR}\left(75.47 \mathrm{MHz}, \mathrm{CDCl}_{3}\right)$ $\delta 21.6\left(-\mathrm{CH}_{3}\right), 33.2\left(-\mathrm{CH}_{2},{ }^{2} J_{P C}=2.8 \mathrm{~Hz}\right), 34.3\left(-\mathrm{CH}_{2},{ }^{2} J_{P C}=2.2 \mathrm{~Hz}\right), 40.6\left(\mathrm{P}-\mathrm{CH},{ }^{1} J_{P C}=137.2\right.$ $\mathrm{Hz}), 40.7\left(\mathrm{P}-\mathrm{CH},{ }^{1} J_{P C}=139.4 \mathrm{~Hz}\right), 66.5\left(-\mathrm{OCH},{ }^{3} J_{P C}=14.2 \mathrm{~Hz}\right), 67.3\left(-\mathrm{OCH},{ }^{3} J_{P C}=9.9 \mathrm{~Hz}\right), 67.6$ $\left(-\mathrm{OCH}_{2},{ }^{2} J_{P C}=7.2 \mathrm{~Hz}\right), 67.7\left(-\mathrm{OCH}_{2},{ }^{2} J_{P C}=7.1 \mathrm{~Hz}\right), 68.2\left(-\mathrm{OCH}_{2},{ }^{2} J_{P C}=7.1 \mathrm{~Hz}\right), 68.3\left(-\mathrm{OCH}_{2}\right.$, $\left.{ }^{2} J_{P C}=6.6 \mathrm{~Hz}\right), 72.8\left(-\mathrm{OCH}_{2}\right), 73.7\left(-\mathrm{OCH}_{2}\right), 127.6-130.0(=\mathrm{CH}), 132.6-145.1(=\mathrm{C}) ;{ }^{31} \mathrm{P}$ NMR $\left(121.50 \mathrm{MHz}, \mathrm{CDCl}_{3}\right) \delta 30.0,29.4$; ESMS m/z $581\left([\mathrm{M}]+\mathrm{H}^{+}\right)$.

\section{Dibenzyl 3R,4-epoxybutanephosphonate (5b).}

Was prepared according to the same procedure as 5a yielding a pale yellow oil $(81 \%)$.

$[\alpha]_{\mathrm{D}}=+7.4^{\circ}\left(\mathrm{c}=1.13\right.$ in $\left.\mathrm{CHCl}_{3}\right) ;{ }^{1} \mathrm{H}$ NMR $\left(300.13 \mathrm{MHz}, \mathrm{CDCl}_{3}\right) \delta 1.75-1.93(4 \mathrm{H}, \mathrm{m}), 2.41(1 \mathrm{H}$, $\mathrm{dd}, J=4.8$ and $2.6 \mathrm{~Hz}), 2.68-2.70(1 \mathrm{H}, \mathrm{m}), 2.90(1 \mathrm{H}, \mathrm{ddt}, J=6.1,4.1$ and $2.3 \mathrm{~Hz}), 4.96(1 \mathrm{H}, \mathrm{dd}, J=$ 11.8 and $8.6 \mathrm{~Hz}), 4.96(1 \mathrm{H}, \mathrm{dd}, 11.8$ and $8.1 \mathrm{~Hz}), 5.05(2 \mathrm{H}, \mathrm{dd}, J=11.8$ and $9.0 \mathrm{~Hz}), 7.34(10 \mathrm{H}, \mathrm{m})$;

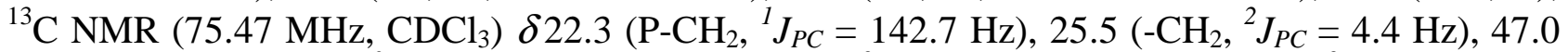
$\left(-\mathrm{OCH}_{2}\right), 51.7\left(-\mathrm{OCH},{ }^{3} J_{P C}=19.8 \mathrm{~Hz}\right), 67.2\left(-\mathrm{OCH}_{2},{ }^{2} J_{P C}=6.6 \mathrm{~Hz}\right), 67.3\left(-\mathrm{OCH}_{2},{ }^{2} J_{P C}=6.5 \mathrm{~Hz}\right)$, $128.0(=\mathrm{CH}), 128.5(=\mathrm{CH}), 128.7(=\mathrm{CH}), 136.3\left(=\mathrm{C},{ }^{3} J_{P C}=6.0 \mathrm{~Hz}\right) ;{ }^{31} \mathrm{P} \mathrm{NMR}(121.50 \mathrm{MHz}$, $\left.\mathrm{CDCl}_{3}\right) \delta 32.2 ; \mathrm{ESMS} m / z 333\left([\mathrm{M}]+\mathrm{H}^{+}\right), 355\left([\mathrm{M}]+\mathrm{Na}^{+}\right), 687\left([2 \mathrm{M}]+\mathrm{Na}^{+}\right)$.

\section{Dibenzyl 1-phenyl-3,4-epoxybutanephosphonate (6).}

Was prepared according to the same procedure of $\mathbf{5 a}$ yielding a colorless oil (89\%).

${ }^{1} \mathrm{H}$ NMR $\left(300.13 \mathrm{MHz}, \mathrm{CDCl}_{3}\right) \delta 1.99-2.33(4 \mathrm{H}, \mathrm{m}), 2.34(1 \mathrm{H}, \mathrm{dd}, J=4.9$ and $2.6 \mathrm{~Hz}), 2.37(1 \mathrm{H}$, $\mathrm{dd}, J=5.1$ and $2.6 \mathrm{~Hz}), 2.58-2.61(2 \mathrm{H}, \mathrm{m}), 2.66-2.73(1 \mathrm{H}, \mathrm{m}), 2.85-2.91(1 \mathrm{H}, \mathrm{m}), 3.25(1 \mathrm{H}, \mathrm{ddd}, J$ $=22.5,9.0$ and $6.2 \mathrm{~Hz}), 3.35(1 \mathrm{H}$, ddd, $J=22.6,11.8$ and $3.9 \mathrm{~Hz}), 4.57(1 \mathrm{H}, \mathrm{dd}, J=11.7$ and 8.3 $\mathrm{Hz}), 4.59(1 \mathrm{H}, \mathrm{dd}, J=11.7$ and $8.4 \mathrm{~Hz}), 4.78(1 \mathrm{H}, \mathrm{dd}, J=11.7$ and $7.2 \mathrm{~Hz}), 4.79(1 \mathrm{H}, \mathrm{dd}, J=11.8$ and $7.1 \mathrm{~Hz}), 4.91(1 \mathrm{H}, \mathrm{dd}, J=11.8$ and $7.7 \mathrm{~Hz}), 4.92(1 \mathrm{H}, \mathrm{dd}, J=11.8$ and $7.8 \mathrm{~Hz}), 4.99(1 \mathrm{H}, \mathrm{dd}, J$ $=11.8$ and $9.3 \mathrm{~Hz}), 5.00(1 \mathrm{H}, \mathrm{dd}, J=11.7$ and $9.3 \mathrm{~Hz}), 7.05-7.40(30 \mathrm{H}, \mathrm{m}) ;{ }^{13} \mathrm{C} \mathrm{NMR}(75.47 \mathrm{MHz}$, 
$\left.\mathrm{CDCl}_{3}\right) \delta 33.2\left(-\mathrm{CH}_{2},{ }^{2} J_{P C}=2.7 \mathrm{~Hz}\right), 33.3\left(-\mathrm{CH}_{2},{ }^{2} J_{P C}=2.8 \mathrm{~Hz}\right), 42.1\left(\mathrm{P}-\mathrm{CH},{ }^{1} J_{P C}=139.4 \mathrm{~Hz}\right)$, $42.2\left(\mathrm{P}-\mathrm{CH},{ }^{1} J_{P C}=137.7 \mathrm{~Hz}\right), 47.1\left(-\mathrm{OCH}_{2}\right), 47.9\left(-\mathrm{OCH}_{2}\right), 50.1\left(-\mathrm{OCH},{ }^{3} J_{P C}=19.7 \mathrm{~Hz}\right), 50.8$ $\left(-\mathrm{OCH},{ }^{3} J_{P C}=15.9 \mathrm{~Hz}\right), 67.5\left(-\mathrm{OCH}_{2},{ }^{2} J_{P C}=7.1 \mathrm{~Hz}\right), 68.2\left(-\mathrm{OCH}_{2},{ }^{2} J_{P C}=6.0 \mathrm{~Hz}\right), 127.5-129.9$ $(=\mathrm{CH}), 135.0\left(=\mathrm{C}, J_{P C}=6.6 \mathrm{~Hz}\right), 135.6\left(=\mathrm{C}, J_{P C}=7.7 \mathrm{~Hz}\right), 136.3\left(=\mathrm{C}, J_{P C}=6.0 \mathrm{~Hz}\right) ;{ }^{31} \mathrm{P}$ NMR $\left(121.50 \mathrm{MHz}, \mathrm{CDCl}_{3}\right) \delta 28.8,28.9 ;$ ESMS m/z $409\left([\mathrm{M}]+\mathrm{H}^{+}\right)$.

(1R,2S)-Dibenzyl 2-(hydroxymethyl)cyclopropylphosphonate (10b).

Was prepared according to the same procedure as $\mathbf{1 0 a}$ but was crystallised in pentane/tert-butyl methyl ether giving white crystals $(39 \%)$.

mp $57{ }^{\circ} \mathrm{C} ;[\alpha]_{\mathrm{D}}=-15.8^{\circ}\left(\mathrm{c}=1.2\right.$ in $\left.\mathrm{CHCl}_{3}\right) ;{ }^{1} \mathrm{H}$ NMR $\left(\mathrm{C}_{6} \mathrm{D}_{6}, 300.13 \mathrm{MHz}\right) \delta 0.46(1 \mathrm{H}$, dddd, $J=$ 11.6, 9.2, 5.7 and $4.4 \mathrm{~Hz}), 0.74(1 \mathrm{H}$, dddd, $J=9.2,5.5,5.5$ and $5.2 \mathrm{~Hz}), 1.10(1 \mathrm{H}, \mathrm{dddd}, J=17.7$, 8.4, 5.9 and $4.4 \mathrm{~Hz}), 1.69$ (1H, dddddd, $J=16.0,8.4,6.4,5.4,5.4$ and $5.4 \mathrm{~Hz}), 1.79(1 \mathrm{H}$, br s), 3.03 $(1 \mathrm{H}, \mathrm{dd}, J=11.5$ and $6.5 \mathrm{~Hz}), 3.35(1 \mathrm{H}, \mathrm{ddd}, J=11.5,5.4$ and $2.0 \mathrm{~Hz}), 4.89(1 \mathrm{H}, \mathrm{dd}, J=11.8$ and $8.5 \mathrm{~Hz}), 4.95(1 \mathrm{H}, \mathrm{dd}, J=11.8$ and $8.5 \mathrm{~Hz}), 4.96(1 \mathrm{H}, \mathrm{dd}, J=11.9$ and $8.5 \mathrm{~Hz}), 5.04(1 \mathrm{H}, \mathrm{dd}, J=$ 12.2 and $8.5 \mathrm{~Hz}), 7.00-7.27(10 \mathrm{H}, \mathrm{m}) ;{ }^{13} \mathrm{C} \mathrm{NMR}\left(75.47 \mathrm{MHz}, \mathrm{C}_{6} \mathrm{D}_{6}\right) \delta 8.3\left(-\mathrm{CH}_{2},{ }^{2} J_{P C}=5.5 \mathrm{~Hz}\right)$, $9.4\left(\mathrm{P}-\mathrm{CH},{ }^{1} J_{P C}=195.9 \mathrm{~Hz}\right), 19.7\left(-\mathrm{CH},{ }^{2} J_{P C}=4.4 \mathrm{~Hz}\right), 64.8\left(-\mathrm{OCH}_{2},{ }^{3} J_{P C}=3.8 \mathrm{~Hz}\right), 67.6\left(-\mathrm{OCH}_{2}\right.$, $\left.{ }^{2} J_{P C}=6.1 \mathrm{~Hz}\right), 67.7\left(-\mathrm{OCH}_{2},{ }^{2} J_{P C}=6.5 \mathrm{~Hz}\right), 128.1(=\mathrm{CH}), 128.6(=\mathrm{CH}), 128.8(=\mathrm{CH}), 136.6(=\mathrm{C})$; ${ }^{31} \mathrm{P}$ NMR $\left(121.50 \mathrm{MHz}, \mathrm{C}_{6} \mathrm{D}_{6}\right) \delta 45.1$; ESMS $m / z 333\left([\mathrm{M}]+\mathrm{H}^{+}\right), 665\left([2 \mathrm{M}]+\mathrm{H}^{+}\right), 687\left(\left[2 \mathrm{M}^{+}+\mathrm{Na}^{+}\right)\right.$.

\section{Dibenzyl 2-(hydroxymethyl)-1-phenylcyclopropylphosphonate (17).}

Was prepared according to the same procedure as $\mathbf{1 0 a}$ yielding a thick grey-white oil. (52\%).

${ }^{1} \mathrm{H}$ NMR (300.13 MHz, $\left.\mathrm{CDCl}_{3}\right) \delta 1.25(1 \mathrm{H}$, br s), $1.38(1 \mathrm{H}$, ddd, $J=9.0,8.8$ and $4.8 \mathrm{~Hz}), 1.65(1 \mathrm{H}$, ddd, $J=16.9 \mathrm{~Hz}, 6.7 \mathrm{~Hz}$ and $4.8 \mathrm{~Hz}), 1.77(1 \mathrm{H}$, ddddd, 11.8, 9.2, 8.8, 6.7 and $4.4 \mathrm{~Hz}), 3.79(1 \mathrm{H}$, dd, $J=12.7$ and $9.3 \mathrm{~Hz}), 4.11(1 \mathrm{H}, \mathrm{dd}, J=12.7,4.3$ and $0.9 \mathrm{~Hz}), 4.60(1 \mathrm{H}, \mathrm{dd}, J=11.7$ and $7.9 \mathrm{~Hz})$, $4.79(1 \mathrm{H}, \mathrm{dd}, J=11.7$ and $6.9 \mathrm{~Hz}), 4.93(1 \mathrm{H}, \mathrm{dd}, J=11.7$ and $7.7 \mathrm{~Hz}), 5.05(1 \mathrm{H}, \mathrm{dd}, J=11.9$ and 8.5 Hz), 7.10-7.39 (15H, m); ${ }^{13} \mathrm{C}$ NMR (75.47 MHz, $\left.\mathrm{CDCl}_{3}\right) \delta 16.6\left(-\mathrm{CH}_{2},{ }^{2} J_{P C}=2.8 \mathrm{~Hz}\right), 27.4(\mathrm{P}-$ $\left.\mathrm{C},{ }^{1} J_{P C}=187.7 \mathrm{~Hz}\right), 29.7\left(-\mathrm{CH},{ }^{2} J_{P C}=1.1 \mathrm{~Hz}\right), 61.4\left(-\mathrm{OCH}_{2},{ }^{3} J_{P C}=7.1 \mathrm{~Hz}\right), 67.7\left(-\mathrm{OCH}_{2},{ }^{2} J_{P C}=\right.$ $6.6 \mathrm{~Hz}), 68.1\left(-\mathrm{OCH}_{2},{ }^{2} J_{P C}=7.1 \mathrm{~Hz}\right), 127.5(=\mathrm{CH}), 127.7(=\mathrm{CH}), 128.0(=\mathrm{CH}), 128.4(=\mathrm{CH}), 128.5$ $(=\mathrm{CH}), 128.6(=\mathrm{CH}), 131.1(=\mathrm{CH}), 131.2(=\mathrm{CH}), 136.1\left(=\mathrm{C},{ }^{3} J_{P C}=6.0 \mathrm{~Hz}\right), 136.1\left(=\mathrm{C},{ }^{3} J_{P C}=6.6\right.$ $\mathrm{Hz}), 139.0\left(=\mathrm{C},{ }^{2} J_{P C}=2.2 \mathrm{~Hz}\right) ;{ }^{31} \mathrm{P}$ NMR $\left(121.50 \mathrm{MHz}, \mathrm{CDCl}_{3}\right) \delta 28.2 ; \mathrm{ESMS} m / z 409\left([\mathrm{M}]+\mathrm{H}^{+}\right)$.

\section{$(1 R, 2 S)$-Dibenzyl 2-(p-toluenesulfonyloxymethyl)cyclopropylphosphonate (11b).}

Was prepared according to the same procedure as 11a but was additionaly crystallised in pentane/ tert-butyl methyl ether yielding white cotton-like crystals $(85 \%)$.

ee\%: > 99\% (hexane/EtOH: $9 / 1$ isocratic).

mp 61-63 ${ }^{\circ} \mathrm{C} ;[\alpha]_{\mathrm{D}}=-17.0^{\circ}\left(\mathrm{c}=1.12\right.$ in $\left.\mathrm{CHCl}_{3}\right) ;{ }^{1} \mathrm{H}$ NMR $\left(300.13 \mathrm{MHz}, \mathrm{CDCl}_{3}\right) \delta 0.60-0.82(2 \mathrm{H}$, $\mathrm{m}), 0.96-1.08(1 \mathrm{H}, \mathrm{m}), 1.68(1 \mathrm{H}$, dddddd, $J=15.2,8.4,7.0,6.8,5.4$ and $5.4 \mathrm{~Hz}), 2.34(3 \mathrm{H}, \mathrm{s}), 3.67$ $(1 \mathrm{H}, \mathrm{dd}, J=10.5$ and $7.0 \mathrm{~Hz}), 3.81(1 \mathrm{H}, \mathrm{ddd}, J=10.6,6.7$ and $1.6 \mathrm{~Hz}), 4.89(2 \mathrm{H}, \mathrm{dd}, J=11.9$ and $8.4 \mathrm{~Hz}), 4.95(1 \mathrm{H}, \mathrm{dd}, J=11.4$ and $8.4 \mathrm{~Hz}), 4.96(1 \mathrm{H}, \mathrm{dd}, J=11.4$ and $8.3 \mathrm{~Hz}), 7.22(12 \mathrm{H}, \mathrm{m}), 7.65$ $(2 \mathrm{H}, \mathrm{d}, J=8.3 \mathrm{~Hz}) ;{ }^{13} \mathrm{C} \mathrm{NMR}\left(75.47 \mathrm{MHz}, \mathrm{CDCl}_{3}\right) \delta 8.7\left(-\mathrm{CH}_{2},{ }^{2} J_{P C}=4.9 \mathrm{~Hz}\right), 10.1\left(\mathrm{P}-\mathrm{CH},{ }^{1} J_{P C}=\right.$ $195.9 \mathrm{~Hz}), 15.9\left(-\mathrm{CH},{ }^{2} J_{P C}=3.9 \mathrm{~Hz}\right), 21.6\left(-\mathrm{CH}_{3}\right), 67.5\left(-\mathrm{OCH}_{2},{ }^{2} J_{P C}=6.1 \mathrm{~Hz}\right), 67.7\left(-\mathrm{OCH}_{2},{ }^{2} J_{P C}\right.$ $=6.1 \mathrm{~Hz}), 72.1\left(-\mathrm{OCH}_{2},{ }^{3} J_{P C}=4.0 \mathrm{~Hz}\right), 127.9(=\mathrm{CH}), 128.5(=\mathrm{CH}), 128.6(=\mathrm{CH}), 129.9(=\mathrm{CH})$, $133.0(=\mathrm{C}), 136.2\left(=\mathrm{C},{ }^{3} J_{P C}=6.1 \mathrm{~Hz}\right), 136.3\left(=\mathrm{C},{ }^{3} J_{P C}=6.0 \mathrm{~Hz}\right), 145.0(\mathrm{C}=) ;{ }^{31} \mathrm{P}$ NMR $(121.50$ $\left.\mathrm{MHz}, \mathrm{CDCl}_{3}\right) \delta 29.0 ; \mathrm{ESMS} m / z 487\left([\mathrm{M}]+\mathrm{H}^{+}\right)$.

\section{Dibenzyl 2-(p-toluenesulfonyloxymethyl)-1-phenylcyclopropylphosphonate (18).}

Was prepared according to the same procedure as 11a yielding a thick grey-white oil. (74\%). 
${ }^{1} \mathrm{H}$ NMR (300.13 MHz, $\left.\mathrm{CDCl}_{3}\right) \delta 1.42(1 \mathrm{H}, \mathrm{ddd}, J=9.2,9.2$ and $4.9 \mathrm{~Hz}), 1.58(1 \mathrm{H}, \mathrm{ddd}, J=17.2$, 6.5 and $4.8 \mathrm{~Hz}), 1.87(1 \mathrm{H}$, ddddd, $J=11.8,8.6,8.6,6.8$ and $6.8 \mathrm{~Hz}), 2.44(3 \mathrm{H}, \mathrm{s}), 4.66(1 \mathrm{H}, \mathrm{ddd}, J$ $=10.8,7.0$ and $1.1 \mathrm{~Hz}), 4.71(1 \mathrm{H}, \mathrm{dd}, J=10.8$ and $8.6 \mathrm{~Hz}), 4.87(1 \mathrm{H}, \mathrm{dd}, J=12.1$ and $8.6 \mathrm{~Hz}), 4.94$ $(1 \mathrm{H}, \mathrm{dd}, J=12.1$ and $7.3 \mathrm{~Hz}), 4.99(1 \mathrm{H}, \mathrm{dd}, J=12.1$ and $8.1 \mathrm{~Hz}), 5.00(1 \mathrm{H}, \mathrm{dd}, J=11.6$ and 7.7 $\mathrm{Hz}), 7.19-7.34(17 \mathrm{H}, \mathrm{m}), 7.81(2 \mathrm{H}, \mathrm{d}, J=8.3 \mathrm{~Hz}) ;{ }^{13} \mathrm{C} \mathrm{NMR}\left(75.47 \mathrm{MHz}, \mathrm{CDCl}_{3}\right) \delta 16.3\left(-\mathrm{CH}_{2}\right.$, $\left.{ }^{2} J_{P C}=1.1 \mathrm{~Hz}\right), 21.6\left(-\mathrm{CH}_{3}\right), 25.8\left(-\mathrm{CH},{ }^{2} J_{P C}=2.2 \mathrm{~Hz}\right), 27.1\left(\mathrm{P}-\mathrm{C},{ }^{1} J_{P C}=188.3 \mathrm{~Hz}\right), 67.7\left(-\mathrm{OCH}_{2}\right.$, $\left.{ }^{2} J_{P C}=6.1 \mathrm{~Hz}\right), 67.8\left(-\mathrm{OCH}_{2},{ }^{2} J_{P C}=6.6 \mathrm{~Hz}\right), 70.3\left(-\mathrm{OCH}_{2},{ }^{3} J_{P C}=5.4 \mathrm{~Hz}\right), 127.6(=\mathrm{CH}), 127.7$ $(=\mathrm{CH}), 127.9(=\mathrm{CH}), 128.2(=\mathrm{CH}), 128.3(=\mathrm{CH}), 128.5(=\mathrm{CH}), 129.9(=\mathrm{CH}), 131.2(=\mathrm{CH}), 133.5$ $(=\mathrm{C}), 136.3\left(=\mathrm{C},{ }^{3} J_{P C}=5.9 \mathrm{~Hz}\right), 136.4\left(=\mathrm{C},{ }^{3} J_{P C}=6.6 \mathrm{~Hz}\right), 138.3\left(=\mathrm{C}, J_{P C}=1.1 \mathrm{~Hz}\right), 144.6(=\mathrm{C})$; ${ }^{31} \mathrm{P} \mathrm{NMR}\left(121.50 \mathrm{MHz}, \mathrm{CDCl}_{3}\right) \delta 25.9 ; \mathrm{ESMS} m / z, 563\left([\mathrm{M}]+\mathrm{H}^{+}\right)$.

\section{$(1 R, 2 S)$-Dibenzyl}

\section{2-[N-(benzyloxy), $N$-(t-butoxycarbonyl)aminomethyl]-}

\section{cyclopropylphosphonate $(\mathbf{1 2 b})$.}

Was prepared according to the same procedure as 12a yielding a colorless oil (96\%). ee\%: > 99\% (hexane/EtOH: 95/5 isocratic).

$[\alpha]_{\mathrm{D}}=-9.7^{\circ}\left(\mathrm{c}=1.05\right.$ in $\left.\mathrm{CHCl}_{3}\right) ;{ }^{1} \mathrm{H}$ NMR $\left(300.13 \mathrm{MHz}, \mathrm{CDCl}_{3}\right) \delta 0.72-0.89(2 \mathrm{H}, \mathrm{m}), 1.10(1 \mathrm{H}$, dddd, $J=18.2,8.5,5.6$ and $4.2 \mathrm{~Hz}), 1.46(9 \mathrm{H}, \mathrm{s}), 1.73(1 \mathrm{H}$, dddddd, $J=15.7,8.3,6.9,6.9,5.5$ and $5.5 \mathrm{~Hz}), 3.29(2 \mathrm{H}, \mathrm{d}, J=6.7 \mathrm{~Hz}), 4.83(2 \mathrm{H}, \mathrm{s}), 4.97(2 \mathrm{H}, \mathrm{dd}, J=11.8$ and $7.9 \mathrm{~Hz}), 5.03(1 \mathrm{H}, \mathrm{dd}, J=$ 11.9 and $8.3 \mathrm{~Hz}), 5.03(1 \mathrm{H}, \mathrm{dd}, J=12.0$ and $7.9 \mathrm{~Hz}), 7.30-7.38(15 \mathrm{H}, \mathrm{m}) ;{ }^{13} \mathrm{C}$ NMR $(75.47 \mathrm{MHz}$, $\left.\mathrm{CDCl}_{3}\right) \delta 9.3\left(-\mathrm{CH}_{2},{ }^{2} J_{P C}=4.9 \mathrm{~Hz}\right), 10.0\left(\mathrm{P}-\mathrm{CH},{ }^{1} J_{P C}=195.4 \mathrm{~Hz}\right), 15.6\left(-\mathrm{CH},{ }^{2} J_{P C}=3.8 \mathrm{~Hz}\right), 28.3$ $\left(-\mathrm{CH}_{3}\right), 52.8\left(-\mathrm{NCH}_{2},{ }^{3} J_{P C}=4.8 \mathrm{~Hz}\right), 67.3\left(-\mathrm{OCH}_{2},{ }^{2} J_{P C}=6.0 \mathrm{~Hz}\right), 67.4\left(-\mathrm{OCH}_{2},{ }^{2} J_{P C}=5.5 \mathrm{~Hz}\right)$, $77.2\left(-\mathrm{OCH}_{2}\right), 81.6(\mathrm{O}-\mathrm{C}), 127.8(=\mathrm{CH}), 127.9(=\mathrm{CH}), 128.3(=\mathrm{CH}), 128.5(=\mathrm{CH}), 128.6(=\mathrm{CH})$, $129.4(=\mathrm{CH}), 135.6(=\mathrm{C}), 136.5\left(=\mathrm{C},{ }^{3} J_{P C}=6.6 \mathrm{~Hz}\right), 136.6\left(=\mathrm{C},{ }^{3} J_{P C}=6.6 \mathrm{~Hz}\right), 156.6(\mathrm{~N}-\mathrm{C}=\mathrm{O})$; ${ }^{31} \mathrm{P}$ NMR $\left(121.50 \mathrm{MHz}, \mathrm{CDCl}_{3}\right) \delta 30.7$; ESMS $m / z 438\left([\mathrm{M}]-\mathrm{Boc}+2 \mathrm{H}^{+}\right), 538\left([\mathrm{M}]+\mathrm{H}^{+}\right)$

\section{Dibenzyl}

phenylcyclopropylphosphonate (19).

2-[( $N$-(benzyloxy), $N$-( $t$-butoxycarbonyl)aminomethyl]-1-

Was prepared according to the same procedure as 12a yielding a colorless oil (90\%).

${ }^{1} \mathrm{H}$ NMR $\left(300.13 \mathrm{MHz}, \mathrm{CDCl}_{3}\right) \delta 1.34-1.42(1 \mathrm{H}, \mathrm{m}), 1.48(9 \mathrm{H}, \mathrm{s}), 1.63-1.77(2 \mathrm{H}, \mathrm{m}), 3.93(1 \mathrm{H}$, ddd, $J=14.8,8.9$ and $1.1 \mathrm{~Hz}), 4.18(1 \mathrm{H}, \mathrm{dd}, J=15.0$ and $3.7 \mathrm{~Hz}), 4.80-4.90(4 \mathrm{H}, \mathrm{m}), 4.93(1 \mathrm{H}$, dd, $J=11.9$ and $8.1 \mathrm{~Hz}) ; 4.99(1 \mathrm{H}, \mathrm{dd}, J=11.9$ and $8.1 \mathrm{~Hz}), 7.26-7.40(20 \mathrm{H}, \mathrm{m}) ;{ }^{13} \mathrm{C} \mathrm{NMR}(75.47$ $\left.\mathrm{MHz}, \mathrm{CDCl}_{3}\right) \delta 18.0\left(-\mathrm{CH}_{2},{ }^{2} J_{P C}=1.1 \mathrm{~Hz}\right), 24.8\left(-\mathrm{CH},{ }^{2} J_{P C}=1.1 \mathrm{~Hz}\right), 26.2\left(\mathrm{P}-\mathrm{C},{ }^{1} J_{P C}=188.3 \mathrm{~Hz}\right)$, $28.3\left(-\mathrm{CH}_{3}\right), 49.2\left(-\mathrm{NCH}_{2},{ }^{3} J_{P C}=5 \mathrm{~Hz}\right), 67.4\left(-\mathrm{OCH}_{2},{ }^{2} J_{P C}=6.6 \mathrm{~Hz}\right), 67.7\left(-\mathrm{OCH}_{2},{ }^{2} J_{P C}=6.0 \mathrm{~Hz}\right)$, $76.8\left(-\mathrm{OCH}_{2}\right), 81.3(\mathrm{O}-\mathrm{C}), 127.2(=\mathrm{CH}), 127.5(=\mathrm{CH}), 127.8(=\mathrm{CH}), 128.1(=\mathrm{CH}), 128.2(=\mathrm{CH})$, $128.3(=\mathrm{CH}), 128.4(=\mathrm{CH}), 129.4(=\mathrm{CH}), 130.0(=\mathrm{CH}), 131.7(=\mathrm{CH}), 135.7(=\mathrm{C}), 136.5\left(=\mathrm{C},{ }^{3} J_{P C}=\right.$ $6.0 \mathrm{~Hz}), 139.3\left(=\mathrm{C},{ }^{2} J_{P C}=1.6 \mathrm{~Hz}\right), 156.5(\mathrm{~N}-\mathrm{C}=\mathrm{O}) ;{ }^{31} \mathrm{P} \mathrm{NMR}\left(121.50 \mathrm{MHz}, \mathrm{CDCl}_{3}\right) \delta 27.3$; ESMS $m / z 514\left([\mathrm{M}]-\mathrm{Boc}+2 \mathrm{H}^{+}\right), 614\left([\mathrm{M}]+\mathrm{H}^{+}\right)$.

$(1 R, 2 S)$-Dibenzyl 2-[N-(benzyloxy)aminomethyl]-cyclopropylphosphonate (13b).

Was prepared according to the same procedure as 13a yielding a pale yellow solid (92\%).

$\operatorname{mp} 40-41{ }^{\circ} \mathrm{C} ;[\alpha]_{\mathrm{D}}=+28.0^{\circ}\left(\mathrm{c}=1.00\right.$ in $\left.\mathrm{CHCl}_{3}\right) ;{ }^{1} \mathrm{H} \mathrm{NMR}\left(300.13 \mathrm{MHz}, \mathrm{CDCl}_{3}\right) \delta 0.61-0.74(2 \mathrm{H}$, $\mathrm{m}), 1.04-1.15(1 \mathrm{H}, \mathrm{m}), 1.60(1 \mathrm{H}$, dddddd, $J=16.1,8.4,6.7,6.7,5.4$ and $5.4 \mathrm{~Hz}) ; 2.66(1 \mathrm{H}, \mathrm{dd}, J=$ 13.2 and $6.8 \mathrm{~Hz}), 2.75(1 \mathrm{H}, \mathrm{ddd}, J=13.2,6.7$ and $1.7 \mathrm{~Hz}), 4.62(2 \mathrm{H}, \mathrm{s}), 4.94(2 \mathrm{H}, \mathrm{dd}, J=11.8$ and $8.1 \mathrm{~Hz}), 5.00(1 \mathrm{H}, \mathrm{dd}, J=11.9$ and $8.3 \mathrm{~Hz}), 5.02(1 \mathrm{H}, \mathrm{dd}, J=11.9$ and $8.3 \mathrm{~Hz}), 7.21-7.31(15 \mathrm{H}, \mathrm{m})$; ${ }^{13} \mathrm{C}$ NMR $\left(75.47 \mathrm{MHz}, \mathrm{CDCl}_{3}\right) \delta 9.3\left(-\mathrm{CH}_{2},{ }^{2} J_{P C}=5.5 \mathrm{~Hz}\right), 10.0\left(\mathrm{P}-\mathrm{CH},{ }^{1} J_{P C}=196.0 \mathrm{~Hz}\right), 15.9$ $\left(-\mathrm{CH},{ }^{2} J_{P C}=3.8 \mathrm{~Hz}\right), 55.3\left(-\mathrm{NCH}_{2},{ }^{3} J_{P C}=3.8 \mathrm{~Hz}\right), 67.3\left(-\mathrm{OCH}_{2},{ }^{2} J_{P C}=6.0 \mathrm{~Hz}\right), 67.4\left(-\mathrm{OCH}_{2},{ }^{2} J_{P C}\right.$ $=6.1 \mathrm{~Hz}), 76.3\left(-\mathrm{OCH}_{2}\right), 127.8(=\mathrm{CH}), 128.3(=\mathrm{CH}), 128.4(=\mathrm{CH}), 128.5(=\mathrm{CH}), 136.6\left(=\mathrm{C},{ }^{3} J_{P C}=\right.$ 
$6.1 \mathrm{~Hz}), 136.6\left(=\mathrm{C},{ }^{3} J_{P C}=6.1 \mathrm{~Hz}\right), 137.8(=\mathrm{C}) ;{ }^{31} \mathrm{P}$ NMR $\left(121.50 \mathrm{MHz}, \mathrm{CDCl}_{3}\right) \delta 31.1 ;$ ESMS $m / z$ $438\left([\mathrm{M}]+\mathrm{H}^{+}\right), 460\left([\mathrm{M}]+\mathrm{Na}^{+}\right), 875\left([2 \mathrm{M}]+\mathrm{H}^{+}\right)$

(1R,2S)-Dibenzyl 2-[ $N$-acetyl, $N$-(benzyloxy)aminomethyl]-cyclopropylphosphonate (14b). Was prepared according to the same procedure as 14a yielding a pale yellow oil (90\%). $[\alpha]_{\mathrm{D}}=+29.0^{\circ}\left(\mathrm{c}=0.2\right.$ in $\left.\mathrm{CHCl}_{3}\right) ;{ }^{1} \mathrm{H}$ NMR $\left(300.13 \mathrm{MHz}, \mathrm{CDCl}_{3}\right) \delta 0.81(1 \mathrm{H}$, dddd, $J=12.0,9.4$, $5.3,4.3 \mathrm{~Hz}), 0.89-0.97(1 \mathrm{H}, \mathrm{m}), 1.11(1 \mathrm{H}$, dddd, $J=18.2,8.4,5.7$ and $4.4 \mathrm{~Hz}), 1.73$ (1H, dddddd, $J$ $=15.7,8.4,7.0,6.8,5.4$ and $5.4 \mathrm{~Hz}), 2.00(3 \mathrm{H}, \mathrm{s}), 3.47(1 \mathrm{H}, \mathrm{dd}, J=14.9$ and $7.4 \mathrm{~Hz}), 3.53(1 \mathrm{H}$, ddd, $J=14.9,6.6$ and $1.8 \mathrm{~Hz}), 4.79(2 \mathrm{H}, \mathrm{s}), 4.95(1 \mathrm{H}, \mathrm{dd}, J=12.1$ and $7.7 \mathrm{~Hz}) ; 4.97(1 \mathrm{H}, \mathrm{dd}, J=$ 11.8 and $8.4 \mathrm{~Hz}), 5.02(1 \mathrm{H}, \mathrm{dd}, J=11.8$ and $8.4 \mathrm{~Hz}), 5.03(1 \mathrm{H}, \mathrm{dd}, J=12.3$ and $8.4 \mathrm{~Hz}), 7.19-7.30$ $(15 \mathrm{H}, \mathrm{m}) ;{ }^{13} \mathrm{C}$ NMR $\left(75.47 \mathrm{MHz}, \mathrm{CDCl}_{3}\right) \delta 9.4\left(-\mathrm{CH}_{2},{ }^{2} J_{P C}=5.0 \mathrm{~Hz}\right), 10.4\left(\mathrm{P}-\mathrm{CH},{ }^{1} J_{P C}=185.4\right.$ $\mathrm{Hz}), 15.8\left(-\mathrm{CH},{ }^{2} J_{P C}=3.8 \mathrm{~Hz}\right), 20.4\left(-\mathrm{CH}_{3}\right), 50.0\left(-\mathrm{NCH}_{2}\right), 67.3\left(-\mathrm{OCH}_{2},{ }^{2} J_{P C}=6.0 \mathrm{~Hz}\right) 67.4$ $\left(-\mathrm{OCH}_{2},{ }^{2} J_{P C}=6.0 \mathrm{~Hz}\right), 76.9\left(-\mathrm{OCH}_{2}\right), 127.7(=\mathrm{CH}), 127.8(=\mathrm{CH}), 128.3(=\mathrm{CH}), 128.5(=\mathrm{CH})$, $128.7(=\mathrm{CH}), 129.0(=\mathrm{CH}), 129.1(=\mathrm{CH}), 134.4(=\mathrm{C}), 136.5\left(=\mathrm{C},{ }^{3} J_{P C}=6.1 \mathrm{~Hz}\right), 136.6\left(=\mathrm{C},{ }^{3} J_{P C}=\right.$ $6.6 \mathrm{~Hz}), 167.8(\mathrm{~N}-\mathrm{C}=\mathrm{O}) ;{ }^{31} \mathrm{P} \mathrm{NMR}\left(121.50 \mathrm{MHz}, \mathrm{CDCl}_{3}\right) \delta 30.5 ; \mathrm{ESMS} m / z .480\left([\mathrm{M}]+\mathrm{H}^{+}\right)$

\section{Dibenzyl 2-[N-(acetyl), $N$-(benzyloxy)aminomethyl]-1-phenylcyclopropylphosphonate (21).}

Was prepared using the same procedure as described for 13a/14a yielding a pale yellow oil $(67 \%$ yield from 19).

${ }^{1} \mathrm{H}$ NMR (300.13 MHz, $\left.\mathrm{CDCl}_{3}\right) \delta 1.28(1 \mathrm{H}, \mathrm{ddd}, J=8.7,8.7$ and $4.5 \mathrm{~Hz}), 1.58(1 \mathrm{H}$, ddd, $J=16.9$, 6.6 and $4.6 \mathrm{~Hz}), 1.73(1 \mathrm{H}$, ddddd, $J=11.9,8.5,8.5,6.6$ and $5.4 \mathrm{~Hz}), 1.93(3 \mathrm{H}, \mathrm{s}), 3.96(1 \mathrm{H}, \mathrm{dd}, J=$ 14.9 and $8.7 \mathrm{~Hz}), 4.35-4.39(1 \mathrm{H}, \mathrm{m}), 4.66-4.76(4 \mathrm{H}, \mathrm{m}), 4.83(1 \mathrm{H}, \mathrm{dd}, J=11.8$ and $8.3 \mathrm{~Hz}), 4.92$ $(1 \mathrm{H}, \mathrm{dd}, J=11.8$ and $8.2 \mathrm{~Hz}), 7.05-7.25(20 \mathrm{H}, \mathrm{m}) ;{ }^{13} \mathrm{C} \mathrm{NMR}\left(75.47 \mathrm{MHz}, \mathrm{CDCl}_{3}\right) \delta 17.7\left(-\mathrm{CH}_{2}\right.$, $\left.{ }^{2} J_{P C}=1.1 \mathrm{~Hz}\right), 20.7\left(-\mathrm{CH}_{3}\right), 24.9\left(-\mathrm{CH},{ }^{2} J_{P C}=2.2 \mathrm{~Hz}\right), 26.3\left(\mathrm{P}-\mathrm{C},{ }^{1} J_{P C}=188.3 \mathrm{~Hz}\right), 45.1\left(-\mathrm{NCH}_{2}\right)$, $67.5\left(-\mathrm{OCH}_{2},{ }^{2} J_{P C}=6.6 \mathrm{~Hz}\right), 67.9\left(-\mathrm{OCH}_{2},{ }^{2} J_{P C}=6.6 \mathrm{~Hz}\right), 76.2\left(-\mathrm{OCH}_{2}\right), 127.3(=\mathrm{CH}), 127.6$ $(=\mathrm{CH}), 127.9(=\mathrm{CH}), 128.1(=\mathrm{CH}), 128.3(=\mathrm{CH}), 128.4(=\mathrm{CH}), 128.6(=\mathrm{CH}), 128.8(=\mathrm{CH}), 129.4$ $(=\mathrm{CH}), 131.1(=\mathrm{CH}), 134.6(=\mathrm{C}), 136.4\left(=\mathrm{C},{ }^{3} J_{P C}=7.1 \mathrm{~Hz}\right), 136.4\left(=\mathrm{C},{ }^{3} J_{P C}=5.7 \mathrm{~Hz}\right), 139.0(=\mathrm{C}$, $\left.{ }^{2} J_{P C}=1.6 \mathrm{~Hz}\right), 169.2(\mathrm{~N}-\mathrm{C}=\mathrm{O}) ;{ }^{31} \mathrm{P} \mathrm{NMR}\left(121.50 \mathrm{MHz}, \mathrm{CDCl}_{3}\right) \delta 27.2 ; \mathrm{ESMS} m / z 556\left([\mathrm{M}]+\mathrm{H}^{+}\right)$

(1R,2S)-Dibenzyl 2-[ $N$-(benzyloxy, $N$-(formyl)aminomethyl]-cyclopropylphosphonate (14c).

To a solution of $85 \%$ formic acid $(269 \mu \mathrm{L}, 4.98 \mathrm{mmol})$ in $\mathrm{CHCl}_{3}(2.5 \mathrm{~mL})$ was added dropwise a solution of DCC $(513 \mathrm{mg}, 2.487 \mathrm{mmol})$ in $\mathrm{CHCl}_{3}(3 \mathrm{~mL})$ at $0{ }^{\circ} \mathrm{C}$ and the reaction was stirred for 5 min at $0{ }^{\circ} \mathrm{C}$. This mixture was then added to a solution of crude amine $13 \mathbf{b}(544 \mathrm{mg})$ in pyridine $(3$ $\mathrm{mL}$ ) dropwise to a solution of solution at $0{ }^{\circ} \mathrm{C}$ and the reaction mixture was stirred for $2 \mathrm{~h}$ at $0{ }^{\circ} \mathrm{C}$ and overnight at $\mathrm{rt}$. After evaporation of the mixture under reduced pressure $\mathrm{Et}_{2} \mathrm{O}(20 \mathrm{~mL})$ was added, the solids were filtered and washed with $\mathrm{Et}_{2} \mathrm{O}(20 \mathrm{~mL})$. The filtrate was evaporated under reduced pressure and the excess pyridine was removed via azeotropic distillation with toluene. A pentane/ $\mathrm{CH}_{2} \mathrm{Cl}_{2}$ /acetone: $1 / 1 / 1$ mixture $(10 \mathrm{~mL})$ was then added, the solids were filtered and the filtrate was evaporated under reduced pressure. $\mathrm{Et}_{2} \mathrm{O}$ was added, the solids were filtered and the filtrate was evaporated to give $350 \mathrm{mg}$ of yellowish oil in a yield of $60 \%$.

$[\alpha]_{\mathrm{D}}=-16.3\left(\mathrm{c}=1.02\right.$ in $\left.\mathrm{CHCl}_{3}\right) ;{ }^{1} \mathrm{H}$ NMR $\left(300.13 \mathrm{MHz}, \mathrm{CDCl}_{3}\right) \delta 0.69-0.96(2 \mathrm{H}, \mathrm{m}), 1.14(1 \mathrm{H}$, dddd, $J=18.2,8.6 ; 5.5$ and $4.3 \mathrm{~Hz}), 1.64-1.76(1 \mathrm{H}, \mathrm{m}), 3.41(2 \mathrm{H}, \mathrm{m}), 4.81(2 \mathrm{H}, \mathrm{s}), 4.98(1 \mathrm{H}, \mathrm{dd}, J$ $=11.9$ and $8.2 \mathrm{~Hz}), 4.99(1 \mathrm{H}, \mathrm{dd}, J=12.0$ and $8.1 \mathrm{~Hz}), 5.04(1 \mathrm{H}, \mathrm{dd}, J=12.0$ and $8.6 \mathrm{~Hz}), 5.05$ $(1 \mathrm{H}, \mathrm{dd}, J=11.8$ and $8.5 \mathrm{~Hz}), 7.31-7.33(15 \mathrm{H}, \mathrm{m}), 8.11(1 \mathrm{H}, \mathrm{s}) ;{ }^{13} \mathrm{C} \mathrm{NMR}\left(75.47 \mathrm{MHz}, \mathrm{CDCl}_{3}\right)$ $\delta 9.3\left(-\mathrm{CH}_{2},{ }^{2} J_{P C}=5.0 \mathrm{~Hz}\right), 10.4\left(\mathrm{P}-\mathrm{CH},{ }^{1} J_{P C}=195.4 \mathrm{~Hz}\right), 15.4(-\mathrm{CH}), 47.6\left(-\mathrm{NCH}_{2}\right), 67.4\left(-\mathrm{OCH}_{2}\right.$, $\left.{ }^{2} J_{P C}=6.0 \mathrm{~Hz}\right), 78.3\left(-\mathrm{OCH}_{2}\right), 127.7(=\mathrm{CH}), 127.8(=\mathrm{CH}), 128.3(=\mathrm{CH}), 128.5(=\mathrm{CH}), 128.7(=\mathrm{CH})$, 
$129.1(=\mathrm{CH}), 129.4(=\mathrm{CH}), 134.2(=\mathrm{C}), 136.2\left(=\mathrm{C},{ }^{3} J_{P C}=5.5 \mathrm{~Hz}\right), 136.3\left(=\mathrm{C},{ }^{3} J_{P C}=6.0 \mathrm{~Hz}\right), 163.5$ $(\mathrm{N}-\mathrm{C}=\mathrm{O}) ;{ }^{31} \mathrm{P}$ NMR $\left(121.50 \mathrm{MHz}, \mathrm{CDCl}_{3}\right) \delta 30.3$; ESMS $m / z 466\left([\mathrm{M}]+\mathrm{H}^{+}\right)$.

\section{$(1 R, 2 S)$-Dibenzyl 2-[ $N$-(benzyloxy), $N$-(propionyl)aminomethyl]-cyclopropylphosphonate (14d).}

To a solution of crude amine $\mathbf{1 3 b}(150 \mathrm{mg}, 0.343 \mathrm{mmol})$ in dry pyridine $(2.5 \mathrm{~mL})$ was added dropwise propionic anhydride $(440 \mu \mathrm{L}, 3.25 \mathrm{mmol})$ and the mixture was stirred for $2 \mathrm{~h}$ at $\mathrm{rt}$. The reaction was quenched with a $1 \mathrm{M} \mathrm{HCl}$-solution $(30 \mathrm{~mL})$ and the aqueous layer was extracted three times with $\mathrm{CH}_{2} \mathrm{Cl}_{2}(30 \mathrm{~mL})$. The combined organic fractions were dried on anhydrous $\mathrm{MgSO}_{4}$, filtered and the solvents were removed on rotary evaporation. The residual oil was dissolved in $\mathrm{CH}_{2} \mathrm{Cl}_{2}(50 \mathrm{~mL})$ and the organic phase was washed once with saturated aqueous $\mathrm{NaHCO}_{3}(30 \mathrm{~mL})$. The combined organic phase was dried on anhydrous $\mathrm{MgSO}_{4}$, filtered and the solvents were removed under reduced pressure. The residual oil was purified via column chromatography (pentane/ $\mathrm{CH}_{2} \mathrm{Cl}_{2}$ /acetone: 1/1/1) yielding $168 \mathrm{mg}$ of a pale yellow oil (99\%).

$[\alpha]_{\mathrm{D}}=-5.6^{\circ}\left(\mathrm{c}=1.17\right.$ in $\left.\mathrm{CHCl}_{3}\right) ;{ }^{1} \mathrm{H}$ NMR $\left(300.13 \mathrm{MHz}, \mathrm{CDCl}_{3}\right) \delta 0.75-0.85(1 \mathrm{H}, \mathrm{m}), 0.75-0.96$ $(1 \mathrm{H}, \mathrm{m}), 1.02(3 \mathrm{H}, \mathrm{t}, J=7.7 \mathrm{~Hz}), 1.07-1.16(1 \mathrm{H}, \mathrm{m}), 1.69(1 \mathrm{H}$, dddddd, $J=15.7,8.4,6.8,6.8,5.5$ and $5.5 \mathrm{~Hz}), 2.34(2 \mathrm{H}, \mathrm{q}, J=7.4 \mathrm{~Hz}), 3.50(2 \mathrm{H}, \mathrm{d}, J=7.0 \mathrm{~Hz}), 4.78(2 \mathrm{H}, \mathrm{s}), 4.94(1 \mathrm{H}, \mathrm{dd}, J=11.9$ and $7.5 \mathrm{~Hz}), 4.97(1 \mathrm{H}, \mathrm{dd}, J=12.1$ and $8.1 \mathrm{~Hz}), 5.01(1 \mathrm{H}, \mathrm{dd}, J=12.5$ and $7.6 \mathrm{~Hz}), 5.02(1 \mathrm{H}, \mathrm{dd}, J$ $=12.1$ and $8.2 \mathrm{~Hz}), 7.29-7.38(15 \mathrm{H}, \mathrm{m}) ;{ }^{13} \mathrm{C} \mathrm{NMR}\left(75.47 \mathrm{MHz}, \mathrm{CDCl}_{3}\right) \delta 8.6\left(-\mathrm{CH}_{3}\right), 9.4\left(-\mathrm{CH}_{2}\right.$, $\left.{ }^{2} J_{P C}=4.9 \mathrm{~Hz}\right), 10.2\left(\mathrm{P}-\mathrm{CH},{ }^{l} J_{P C}=195.4 \mathrm{~Hz}\right), 15.7\left(-\mathrm{CH},{ }^{2} J_{P C}=3.9 \mathrm{~Hz}\right), 25.6\left(-\mathrm{CH}_{2}\right), 49.2\left(-\mathrm{NCH}_{2}\right.$, $\left.{ }^{3} J_{P C}=2.2 \mathrm{~Hz}\right), 67.3\left(-\mathrm{OCH}_{2},{ }^{2} J_{P C}=6.0 \mathrm{~Hz}\right), 67.4\left(-\mathrm{OCH}_{2},{ }^{2} J_{P C}=6.0 \mathrm{~Hz}\right), 76.9\left(-\mathrm{OCH}_{2}\right), 127.7$ $(=\mathrm{CH}), 127.8(=\mathrm{CH}), 128.2(=\mathrm{CH}), 128.3(=\mathrm{CH}), 128.5(=\mathrm{CH}), 128.7(=\mathrm{CH}), 128.9(=\mathrm{CH}), 129.0$ $(=\mathrm{CH}), 134.5(=\mathrm{C}), 136.4\left(=\mathrm{C},{ }^{3} J_{P C}=6.6 \mathrm{~Hz}\right), 136.5\left(=\mathrm{C},{ }^{3} J_{P C}=6.6 \mathrm{~Hz}\right), 176.5(\mathrm{~N}-\mathrm{C}=\mathrm{O}) ;{ }^{31} \mathrm{P}$ NMR (121.50 MHz, $\left.\mathrm{CDCl}_{3}\right) \delta 30.7$; ESMS $m / z 494\left([\mathrm{M}]+\mathrm{H}^{+}\right)$.

$(1 R, 2 S)-2-[N$-(acetyl), $N$-(hydroxy)aminomethyl]-cyclopropylphosphonic acid (3b).

Was prepared according to the same procedure as 3a yielding an amber amorphous solid (74\%). $\operatorname{mp} 100-102{ }^{\circ} \mathrm{C} ;[\alpha]_{\mathrm{D}}=-29.5^{\circ}\left(\mathrm{c}=1.23\right.$ in $\left.\mathrm{H}_{2} \mathrm{O}\right) ;{ }^{1} \mathrm{H}$ NMR $(300.13 \mathrm{MHz}, \mathrm{MeOD}) \delta 0.45(1 \mathrm{H}, \mathrm{m})$, $0.57(1 \mathrm{H}, \mathrm{m}), 0.77(1 \mathrm{H}, \mathrm{m}), 1.27(1 \mathrm{H}, \mathrm{m}), 1.95(3 \mathrm{H}, \mathrm{s}), 3.17(1 \mathrm{H}, \mathrm{m}), 3.53(1 \mathrm{H}, \mathrm{dd}, J=14.3$ and 5.3 $\mathrm{Hz}) ;{ }^{13} \mathrm{C}$ NMR $(75.47 \mathrm{MHz}, \mathrm{MeOD}) \delta 9.6\left(-\mathrm{CH}_{2},{ }^{2} J_{P C}=5.1 \mathrm{~Hz}\right), 14.6\left(-\mathrm{CH},{ }^{l} J_{P C}=177.8 \mathrm{~Hz}\right), 15.8$ $\left(-\mathrm{CH},{ }^{2} J_{P C}=6.1 \mathrm{~Hz}\right), 20.2\left(-\mathrm{CH}_{3}\right), 52.7\left(-\mathrm{NCH}_{2},{ }^{3} J_{P C}=3.3 \mathrm{~Hz}\right), 173.6(\mathrm{~N}-\mathrm{C}=\mathrm{O}) ;{ }^{31} \mathrm{P}$ NMR $(121.50$ $\left.\mathrm{MHz}, \mathrm{D}_{2} \mathrm{O}\right) \delta 22.7$; ESMS $m / z 210\left([\mathrm{M}]+\mathrm{H}^{+}\right)$.

$(1 R, 2 S)-2-[N$-(formyl), $N$-(hydroxy)aminomethyl]-cyclopropylphosphonic acid (3c).

Was prepared according to the same procedure as 3a yielding a red amorphous solid (77\%). Elution used: 5 min $5 \mathrm{mM} \mathrm{NH}_{4} \mathrm{OAc}$ solution, 5 min gradient elution to $\mathrm{MeCN}$.

$\operatorname{mp~} 114{ }^{\circ} \mathrm{C} ;[\alpha]_{\mathrm{D}}=-28.6^{\circ}\left(\mathrm{c}=0.32\right.$ in $\left.\mathrm{H}_{2} \mathrm{O}\right) ;{ }^{1} \mathrm{H}$ NMR $\left(300.13 \mathrm{MHz}, \mathrm{D}_{2} \mathrm{O}\right) 0.71(2 \mathrm{H}, \mathrm{m}), \delta 0.85(1 \mathrm{H}$, $\mathrm{m}), 1.37(1 \mathrm{H}, \mathrm{m}), 3.44(2 \mathrm{H}, \mathrm{m}), 7.90(1 \mathrm{H}, \mathrm{br} \mathrm{s}) ;{ }^{13} \mathrm{C} \mathrm{NMR}\left(75.47 \mathrm{MHz}, \mathrm{D}_{2} \mathrm{O}\right) \delta 8.4\left(-\mathrm{CH}_{2},{ }^{2} J_{P C}=\right.$ $3.8 \mathrm{~Hz}), 12.7\left(\mathrm{P}-\mathrm{CH},{ }^{1} J_{P C}=177.3 \mathrm{~Hz}\right), 14.7\left(-\mathrm{CH},{ }^{2} J_{P C}=2.7 \mathrm{~Hz}\right), 54.4\left(-\mathrm{NCH}_{2}\right), 159.5(\mathrm{~N}-\mathrm{C}=\mathrm{O})$; ${ }^{31} \mathrm{P}$ NMR (121.50 MHz, $\left.\mathrm{D}_{2} \mathrm{O}\right) \delta 22.5$; ESMS $m / z .194\left([\mathrm{M}]-\mathrm{H}^{+}\right)$.

$(1 R, 2 S)-2-[N$-(hydroxy), $N$-(propionyl)aminomethyl]-cyclopropylphosphonic acid (3d).

Was prepared according to the same procedure as 3a yielding an amber amorphous solid (86\%). $\operatorname{mp~85-87~}{ }^{\circ} \mathrm{C} ;[\alpha]_{\mathrm{D}}=-30.2^{\circ}\left(\mathrm{c}=1.00\right.$ in $\left.\mathrm{H}_{2} \mathrm{O}\right) ;{ }^{1} \mathrm{H}$ NMR $(300.13 \mathrm{MHz}, \mathrm{MeOD}) \delta 0.56-0.65(1 \mathrm{H}, \mathrm{m})$, 0.68-0.75 (1H, m), 0.85-0.96 (1H, m), $1.09(3 \mathrm{H}, \mathrm{t}, J=7.5 \mathrm{~Hz}), 1.37-1.46(1 \mathrm{H}, \mathrm{m}), 2.49(2 \mathrm{H}, \mathrm{q}, J=$ $7.5 \mathrm{~Hz}), 3.30-3.33(1 \mathrm{H}, \mathrm{m}), 3.69(1 \mathrm{H}, \mathrm{dd}, J=14.3$ and $5.6 \mathrm{~Hz}) ;{ }^{13} \mathrm{C} \mathrm{NMR}(75.47 \mathrm{MHz}, \mathrm{MeOD})$ 
$\delta 9.3\left(-\mathrm{CH}_{3}\right), 9.6\left(-\mathrm{CH}_{2},{ }^{2} J_{P C}=4.9 \mathrm{~Hz}\right), 14.6\left(\mathrm{P}-\mathrm{CH},{ }^{1} J_{P C}=181.1 \mathrm{~Hz}\right), 15.8(-\mathrm{CH}), 26.4\left(-\mathrm{CH}_{2}\right)$, $52.8\left(-\mathrm{NCH}_{2}\right), 176.6(\mathrm{~N}-\mathrm{C}=\mathrm{O}) ;{ }^{31} \mathrm{P} \mathrm{NMR}(121.50 \mathrm{MHz}, \mathrm{MeOD}) \delta 21.6$; ESMS $m / z 224\left([\mathrm{M}]+\mathrm{H}^{+}\right)$

2-[N-(acetyl), $N$-(hydroxy)aminomethyl]-1-phenylcyclopropylphosphonic acid (4).

Was prepared according to the same procedure as 3d yielding light red cotton-like crystals (92\%). An analytical sample was prepared using RP-HPLC (elution used: gradient elution of $5 \mathrm{mM}$ $\mathrm{NH}_{4} \mathrm{OAc}$ to $\mathrm{MeCN}$ in $\left.20 \mathrm{~min}\right)$.

mp $124{ }^{\circ} \mathrm{C} ;{ }^{1} \mathrm{H}$ NMR $\left(300.13 \mathrm{MHz}, \mathrm{D}_{2} \mathrm{O}\right) \delta 1.48-1.57(2 \mathrm{H}, \mathrm{m}), 1.73(1 \mathrm{H}, \mathrm{m}), 2.19(3 \mathrm{H}, \mathrm{s}), 3.99-$ $4.10(1 \mathrm{H}, \mathrm{m}), 4.29(1 \mathrm{H}, \mathrm{dd}, J=14.6$ and $5.0 \mathrm{~Hz}), 7.37-7.51(5 \mathrm{H}, \mathrm{m}) ;{ }^{13} \mathrm{C}$ NMR $\left(75.47 \mathrm{MHz}, \mathrm{D}_{2} \mathrm{O}\right)$ $\delta 16.9\left(-\mathrm{CH}_{2}\right), 19.6(-\mathrm{CH}), 23.9\left(-\mathrm{CH}_{3}\right), 27.6\left(\mathrm{P}-\mathrm{C},{ }^{l} J_{P C}=180.0 \mathrm{~Hz}\right), 38.5\left(-\mathrm{NCH}_{2}\right), 127.6(=\mathrm{CH})$, $128.7(=\mathrm{CH}), 131.1(=\mathrm{CH}), 140.5(=\mathrm{C}), 173.9(\mathrm{~N}-\mathrm{C}=\mathrm{O}) ;{ }^{31} \mathrm{P}$ NMR $\left(121.50 \mathrm{MHz}, \mathrm{D}_{2} \mathrm{O}\right) \delta 23.7$; ESMS $m / z 286\left([\mathrm{M}]+\mathrm{H}^{+}\right), 571\left([2 \mathrm{M}]+\mathrm{H}^{+}\right)$. 


\section{HPLC purity of inhibitors.}

HPLC purity of compounds 3a-d and $\mathbf{4}$ was determined using two different HPLC columns:

1. Phenomenex Luna C-18 (2) $250 \mathrm{~mm}$ x $4.6 \mathrm{~mm}$ (5 $\mu \mathrm{m}$ particle size), detection at $214.20 \mathrm{~nm}$.

2. Alltech Alltima C-8 $250 \mathrm{~mm} \times 4.6 \mathrm{~mm}$ (5 $\mu \mathrm{m}$ particle size), detection at $214.20 \mathrm{~nm}$.

The indicated HPLC conditions (Methods $\mathrm{A}$ and $\mathrm{B}$, Solvent $\mathrm{A}=5 \mathrm{mM} \mathrm{NH} 4 \mathrm{OAc}$ solution, Solvent $\mathrm{B}=\mathrm{MeCN}$, flowrate $=1 \mathrm{~mL} / \mathrm{min}$ ) described below were employed to determine the purity of compounds 3a-d and $\mathbf{4}$

- Method A: 0-2 min: isocratic $100 \%$ A; 2-32 min: gradient 0-20\% B; 32-33 min: gradient $20-100 \%$ B; 33-37 min: isocratic 100\% B.

- Method B: 0-2 min: isocratic $100 \%$ A; 2-32 min: gradient 0-100\% B; 32-37 min: isocratic $100 \%$ B.

\begin{tabular}{lcccc}
\hline Compound & Column & Method & $\mathbf{t}_{\text {ret }}(\mathbf{m i n})$ & Purity (\%) \\
\hline \multirow{3}{*}{ 3a } & 1 & $\mathrm{~A}$ & 5.06 & $96 \%$ \\
\cline { 2 - 5 } & 2 & $\mathrm{~A}$ & 3.21 & $97 \%$ \\
\hline \multirow{3}{*}{ 3b } & 1 & $\mathrm{~A}$ & 5.34 & $98 \%$ \\
\cline { 2 - 5 } & 2 & $\mathrm{~A}$ & 3.23 & $99 \%$ \\
\hline \multirow{3}{*}{ 3d } & 1 & $\mathrm{~B}$ & 3.19 & $99 \%$ \\
\cline { 2 - 5 } & 2 & $\mathrm{~B}$ & 2.48 & $86 \%$ \\
\hline \multirow{4}{*}{$\mathbf{4}$} & 1 & $\mathrm{~B}$ & 8.05 & $94 \%$ \\
\hline & 2 & $\mathrm{~B}$ & 3.45 & $97 \%$ \\
\hline
\end{tabular}

Table 1: HPLC purity of compounds 3a-d and 4 
Determination of relative configuration of cyclopropane rings.

1. Unsubsituted cyclopropane configuration

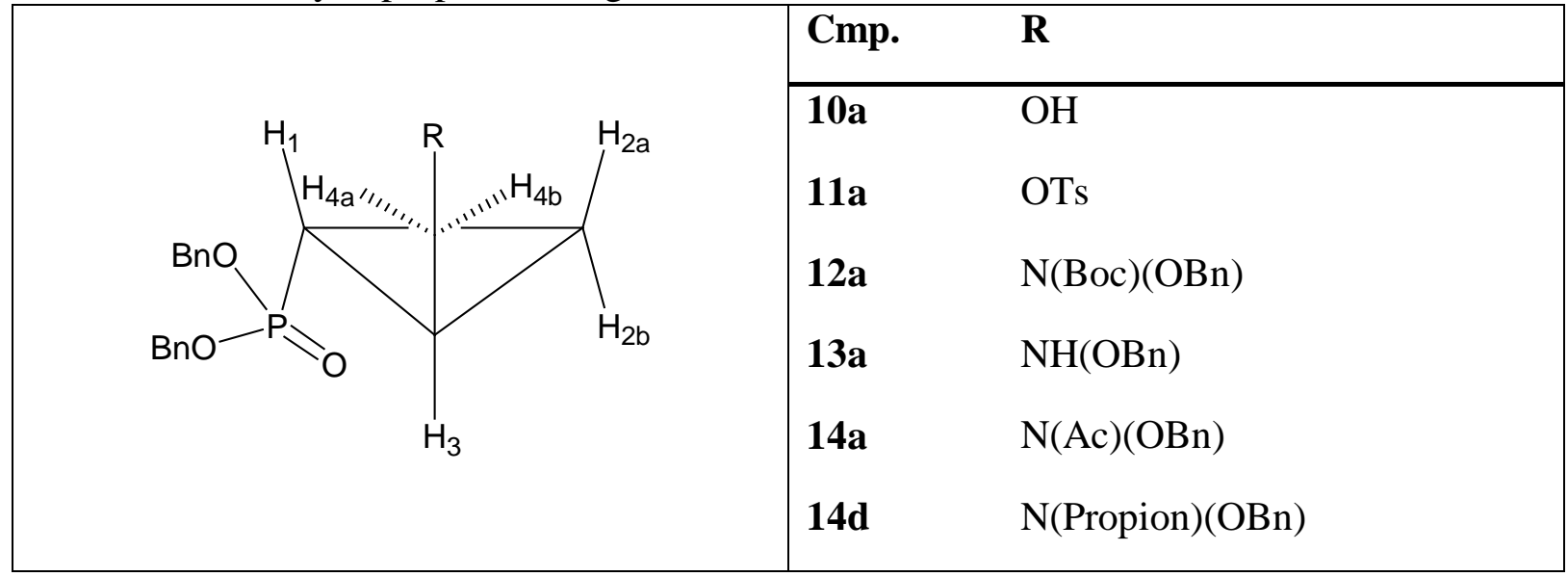

Table 1. Trans-cyclopropane compounds

\begin{tabular}{llllllll} 
Cmp. & $\boldsymbol{\delta}\left(\mathbf{H}_{\mathbf{3}}\right)$ & $\boldsymbol{J}\left(\mathbf{H}_{\mathbf{3}}-\mathbf{H}_{\mathbf{1}}\right)$ & $\boldsymbol{J}\left(\mathbf{H}_{\mathbf{3}}-\mathbf{H}_{\mathbf{2}}\right)$ & $\boldsymbol{J}\left(\mathbf{H}_{\mathbf{3}}-\mathbf{H}_{\mathbf{2}}\right)$ & $\boldsymbol{J}\left(\mathbf{H}_{\mathbf{3}}-\mathbf{H}_{\mathbf{4}}\right)$ & $\boldsymbol{J}\left(\mathbf{H}_{\mathbf{3}}-\mathbf{H}_{\mathbf{4 b}}\right)$ & ${ }^{3} \boldsymbol{J}\left(\mathbf{P}-\mathbf{H}_{\mathbf{3}}\right)$ \\
\hline $\mathbf{1 0 a}$ & 1.69 & $5.4 \mathrm{~Hz}$ & $5.4 \mathrm{~Hz}$ & $8.4 \mathrm{~Hz}$ & $5.4 \mathrm{~Hz}$ & $6.4 \mathrm{~Hz}$ & $16.0 \mathrm{~Hz}$ \\
$\mathbf{1 1 a}$ & 1.68 & $5.4 \mathrm{~Hz}$ & $5.4 \mathrm{~Hz}$ & $8.4 \mathrm{~Hz}$ & $6.8 \mathrm{~Hz}$ & $6.9 \mathrm{~Hz}$ & $15.4 \mathrm{~Hz}$ \\
$\mathbf{1 2 a}$ & 1.73 & $5.5 \mathrm{~Hz}$ & $5.5 \mathrm{~Hz}$ & $8.3 \mathrm{~Hz}$ & $6.9 \mathrm{~Hz}$ & $6.9 \mathrm{~Hz}$ & $15.7 \mathrm{~Hz}$ \\
$\mathbf{1 3 a}$ & 1.60 & $5.4 \mathrm{~Hz}$ & $5.4 \mathrm{~Hz}$ & $8.4 \mathrm{~Hz}$ & $6.7 \mathrm{~Hz}$ & $6.7 \mathrm{~Hz}$ & $16.1 \mathrm{~Hz}$ \\
$\mathbf{1 4 a}$ & 1.73 & $5.4 \mathrm{~Hz}$ & $5.4 \mathrm{~Hz}$ & $8.4 \mathrm{~Hz}$ & $6.8 \mathrm{~Hz}$ & $7.0 \mathrm{~Hz}$ & $15.7 \mathrm{~Hz}$ \\
$\mathbf{1 4 d}$ & 1.69 & $5.5 \mathrm{~Hz}$ & $5.5 \mathrm{~Hz}$ & $8.4 \mathrm{~Hz}$ & $6.8 \mathrm{~Hz}$ & $6.8 \mathrm{~Hz}$ & $15.7 \mathrm{~Hz}$
\end{tabular}

Table 2. Coupling constants of proton $\mathrm{H}_{3}$ signal for trans-cyclopropane compounds

Due to the nonspecific response of NOE experiments the relative configuration of the structure had to be determined using standard ${ }^{1} \mathrm{H}$ NMR coupling constants. Assignment of the hydrogen atoms to the peaks was performed using COSY, ${ }^{13} \mathrm{C}$ and HSQC NMR experiments.

The proton signal of choice for determination is the problematic proton $\mathrm{H}_{3}$. It has an extremely complicated peak pattern due to the vicinity of 5 vicinal hydrogen couplings and an additional $\mathrm{P}-\mathrm{H}$ coupling. 


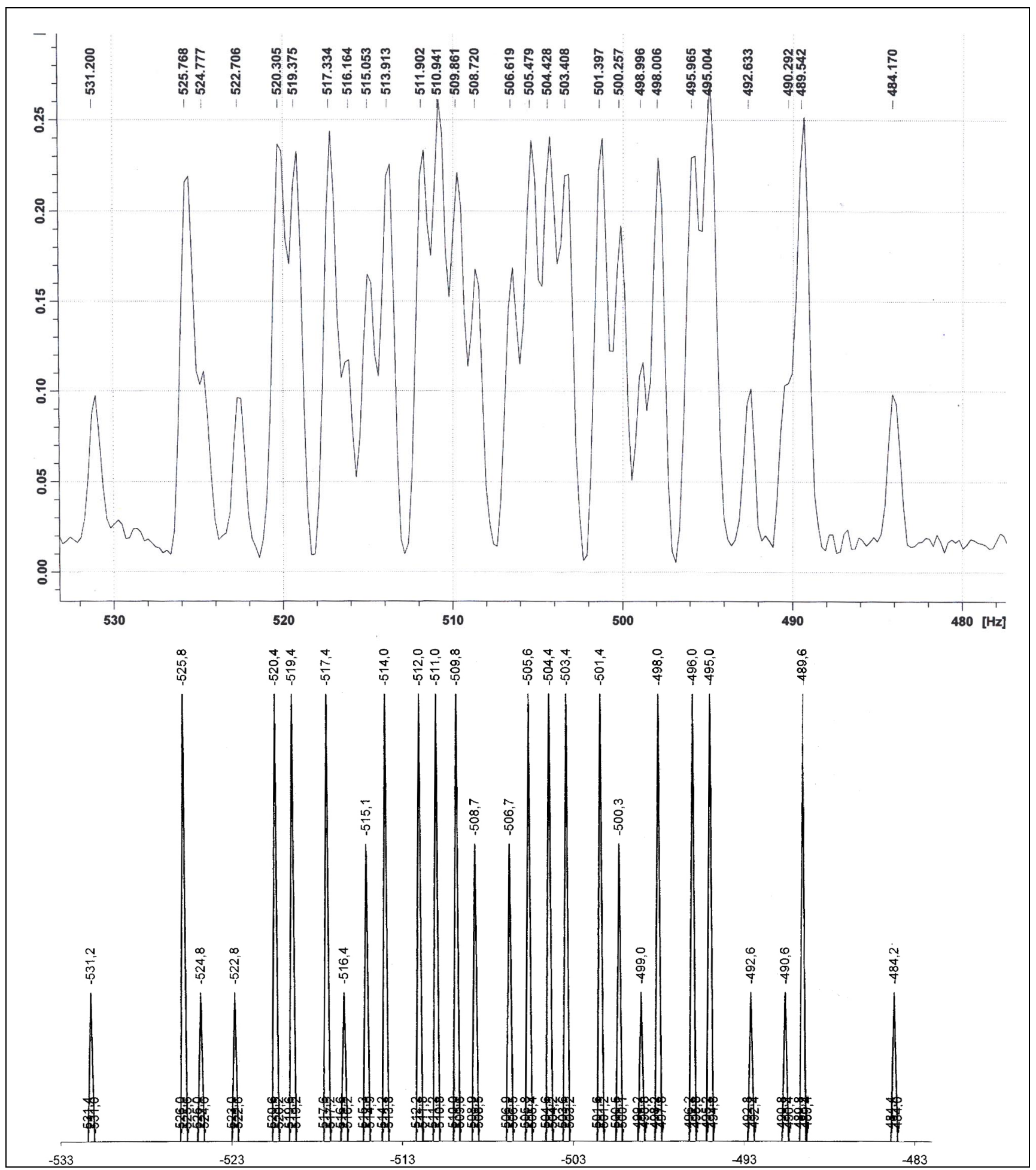

Figure 1. Real spectrum (above) and simulated spectrum (below) of compound 10a 
Using the coupling constants of the vicinal hydrogen atoms $\left(\mathrm{H}_{1}, \mathrm{H}_{2 \mathrm{a}, 2 \mathrm{~b}}, \mathrm{H}_{4 \mathrm{a}, 4 \mathrm{~b}}\right)$ a simulation of the spectrum could be performed. Therefore an Excel spreadsheet was made to calculate the peak intensity and peak pattern. As shown in Figure 1, the simulated spectrum of compound 10a, calculated using the coupling constants of Table 2, shows a remarkable correlation with the real spectrum. Likewise, a peak simulation in Mestre-C, an NMR acquisition and processing software displayed the same correlation.

The trans-configured cyclopropane structure shown in Table $\mathbf{1}$ is the only consistent one based on the coupling constants of proton $\mathrm{H}_{3}$ and other protons. This can be rationalized using these two rules:

1. Coupling constants for cis-proton atoms on a cyclopropane ring are always larger than trans-hydrogen atoms.

2. ${ }^{3} \mathrm{~J}(\mathrm{P}-\mathrm{H})$ coupling constants for cis-configured $\mathrm{P}-\mathrm{H}$ atoms are always greater than trans $\mathrm{P}-\mathrm{H}$ atoms.

Table 2 shows that $J\left(\mathrm{H}_{3}-\mathrm{H}_{2 \mathrm{~b}}\right)$ is larger than $J\left(\mathrm{H}_{3}-\mathrm{H}_{2 \mathrm{a}}\right)$ and $J\left(\mathrm{H}_{3}-\mathrm{H}_{1}\right)$. Moreover, the ${ }^{3} J\left(\mathrm{P}-\mathrm{H}_{3}\right)$ coupling constants are always in a typical range of cis-configured $\mathrm{P}-\mathrm{H}$ atoms.

2. $\alpha$-phenyl substituted cyclopropane configuration

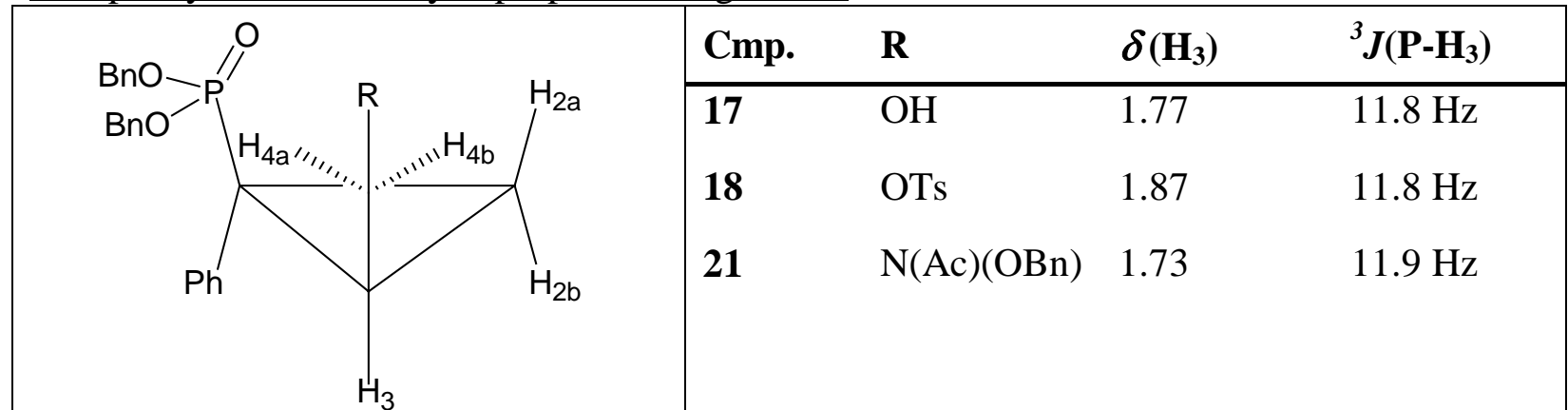

Table 3. Cis-cyclopropane compounds

As shown in Table 3 the ${ }^{3} J\left(\mathrm{P}-\mathrm{H}_{3}\right)$ coupling constants are much lower for the phenylsubstituted cyclopropane ring than for the unsubstituted cyclopropane ring in Table 2 . According to the second rule presented in previous paragraph proton $\mathrm{H}_{3}$ should therefore be oriented trans to the phosphonate.

Additionally we performed a NOESY-experiment on compound 18. The presence of two distinct NOESY correlations, i.e. a correlation between $\mathrm{H}_{4 \mathrm{a}, 4 \mathrm{~b}}$ and $\mathrm{H}_{2 \mathrm{a}}$ and a correlation between a phenyl proton and $\mathrm{H}_{2 b} / \mathrm{H}_{3}$, are indicative for a cis-cyclopropane configuration (Figure 2). Should the cyclopropane ring bear the trans configuration, the phenyl protons would only cause a NOESY correlation with $\mathrm{H}_{2 \mathrm{a}}$ but not with $\mathrm{H}_{2 \mathrm{~b}} / \mathrm{H}_{3}$. 


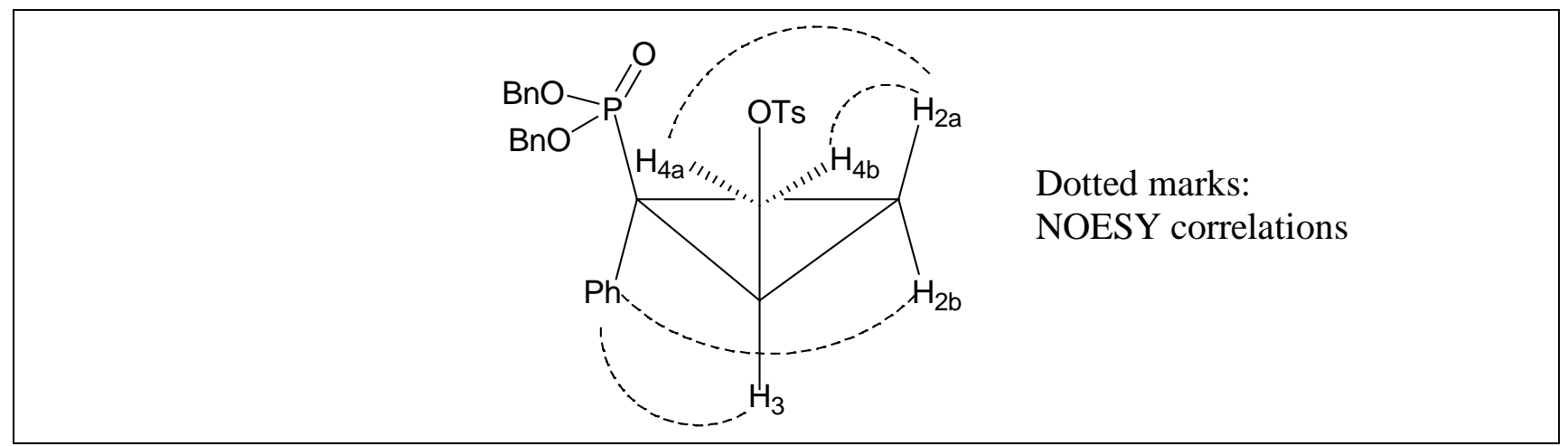

Figure 2. NOESY correlations on cis-cyclopropane compound 18

Finally, the configuration of both the unsubstituted and the phenyl-substituted cyclopropane ring can also be proven via comparison of the ${ }^{3} J(\mathrm{P}-\mathrm{C})$ coupling constants of compounds $10 \mathrm{a}$ and 17. We can observe in Figure 3 that ${ }^{3} J(\mathrm{P}-\mathrm{C})$ of compound 17 is higher than the ${ }^{3} J(\mathrm{P}-\mathrm{C})$ of compound 10a. As reported in literature, ${ }^{3} J(\mathrm{P}-\mathrm{C}$, cis $)$ coupling constants are higher than ${ }^{3} J(\mathrm{P}-\mathrm{C}$, trans $)$ in cyclopropylphosphonates, hence the assignment of trans- and cis-configuration to compounds $\mathbf{1 0 a}$, respectively $17 .^{1,2}$
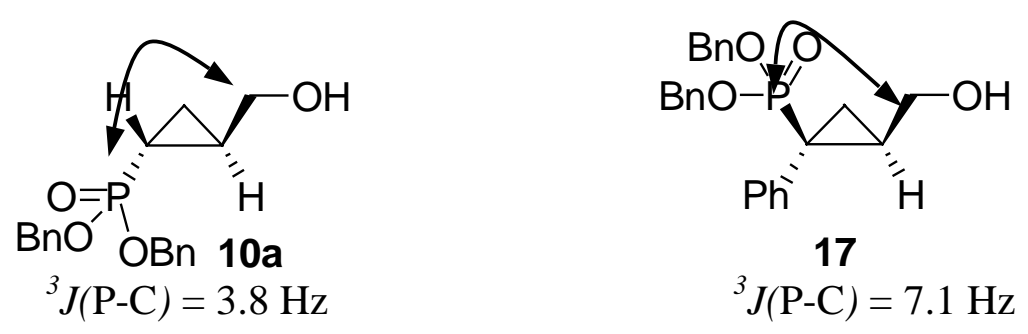

Figure 3. ${ }^{3} J(\mathrm{P}-\mathrm{C})$ coupling constants of $\mathbf{1 0 a}$ and 17.

\section{Referencs}

1. Thiem, J.; Meyer, B. Karplus-type relationship for vicinal coupling J-3(CCCP) in phosphonates. Org. Magn. Reson. 1978, 11, 50-51.

2. Duquenne, C.; Goumain, S.; Jubault, P.; Feasson, C.; Quirion, J.-C. Electrosynthesis of alphaarylated beta-substituted cyclopropylphosphonates. Synthesis of a phosphonic analogue of minalcipran. Org. Lett. 2000, 2, 453-455. 